\title{
Estabilidad de rendimiento y adaptabilidad de híbridos de maíz tolerantes a suelos ácidos en base a las características del análisis GGE biplot Estabilidad y adaptabilidad de híbridos de maíz a suelos ácidos
}

\author{
Carlos Segundo Tirado Soto ${ }^{1 *}$, Víctor Vásquez Arce', Luis Alberto Narro León ${ }^{2}$ \\ ${ }^{1}$ Universidad Nacional de Cajamarca. Cajamarca, Perú. \\ ${ }^{2}$ Centro Internacional de Mejoramiento de Maíz y Trigo-CIMMYT. Cali, Colombia. \\ *Autor para Correspondencia / Corresponding autor, e-mail: catirso@unc.edu.pe
}

\section{Stability of yield and adaptability of corn hybrids tolerant to acid soils based on the characteristics of the GGE biplot analysis}

\begin{abstract}
The main objective of this research was to evaluate the responses of fourteen maize hybrids through twenty-seven test environments to identify high performance genotypes with broad and specific adaptation by applying the GGE (Genotype + Genotype $x$ Environment) biplot analysis. We used the randomized complete block design with three replicates in each locality. Yields were recorded in tons of maize grain per hectare. The GGE biplot analysis revealed that the cultivars G11, G6, G12, G10 and G9 presented a good potential of grain yield and the best stability through all the evaluated environments. Genotypes G4, G8, G9 and G3 presented specific adaptation. The test environments with the most discrimination capacity and most representative and useful for the selection of genotypes with wide adaptation were L5-Pereira and L15-El Líbano. Seven groups of genotypes and environments (mega-environments) were identified, which are areas that show homogeneous environmental conditions and that cause certain genotypes to perform similarly.
\end{abstract}

Keywords: Genotype Environment Interaction, Stability

Editado por /

Edited by:

Gabriela Albán

Recibido /

Received:

2018/04/04

Aceptado /

Accepted:

2018/11/12

Publicado en línea / Published online: 2019/05/20

\section{Resumen}

El objetivo principal de esta investigación fue evaluar las respuestas de catorce híbridos de maíz a través de veintisiete ambientes de prueba para identificar a los genotipos de alto rendimiento con amplia y específica adaptación mediante la aplicación del análisis GGE (Genotipo + Genotipo x Ambiente) biplot. Se utilizó el diseño de bloques completos al azar con tres repeticiones en cada localidad. Se registraron los rendimientos en toneladas de grano de maíz por hectárea. El análisis GGE biplot permitió conocer que los cultivares G11, G6, G12, G10 y G9 presentaron un buen potencial de rendimiento de 
grano y la mejor estabilidad a través de todos los ambientes evaluados. Los genotipos G4, G8, G9 y G3 presentaron adaptación específica. Los ambientes de prueba con mayor capacidad de discriminación y más representativos y útiles para la selección de genotipos con adaptación amplia fueron L5-Pereira y L15-El Líbano. Se identificó siete agrupamientos de genotipos y ambientes (mega-ambientes) que son áreas que muestra condiciones ambientales homogéneas y que causa que ciertos genotipos se desempeñen similarmente.

Palabras claves: Interacción Genotipo Ambiente, Estabilidad

\section{INTRODUCCIÓN}

La demanda de maíz en los países en desarrollo superará al de trigo y arroz para el año 2020, lo que indica la importancia del cultivo en el aspecto nutricional, este requerimiento nos hace mirar con responsabilidad el rol que tienen que desempeñar las instituciones de investigación agrícola del Perú y del mundo para dirigir esfuerzo a satisfacer el rápido crecimiento de la demanda de maíz [1]

Las sabanas de Sudamérica con una superficie de 270 millones de hectáreas representan una de las mayores extensiones con potencial para la producción agrícola [2]. Muchos profesionales que trabajan en el desarrollo del maíz tropical creen que su futuro en esas áreas es muy prometedor y que el aumento de la productividad del maíz, su producción y utilización en los trópicos no son una elección sino una necesidad [3]. La toxicidad por aluminio es el factor limitante más importante para la producción de maíz en los suelos tropicales, ya que está asociado a un bajo pH, alta saturación de aluminio, baja absorción de fósforo, deficiencias de calcio, magnesio fósforo, hierro y molibdeno [4].

Es importante, por lo tanto, desarrollar materiales genéticos que presenten una buena tolerancia a la acidez del suelo y todos los problemas edáficos asociados con ella, ya que ésta es una solución permanente, barata y que eventualmente contribuye a una mejor conservación del ambiente [4]. El Centro Internacional de Mejoramiento de Maíz y Trigo (CIMMYT) viene desarrollando un programa de mejora genética para producir maíces tropicales con tolerancia a suelos ácidos. Entre estos materiales genéticos, el maíz híbrido de grano amarillo duro tiene una gran importancia en el incremento de la productividad y producción del maíz en estas zonas tropicales [2].

En los programas de mejoramiento genético de plantas es común evaluar la respuesta de los genotipos en varios ambientes con el fin de valorar la magnitud y tipo de la interacción genotipo por ambiente (IGE), así como la estabilidad fenotípica de los cultivares sometidos a la totalidad de los ambientes predominantes en una región potencial de adaptación. El entendimiento de la IGE observada en estos ensayos, permite identificar variedades de alto rendimiento con adaptación específica y amplia [5]. Existen varios modelos que nos permiten estudiar la estabilidad.

El Genotipo + Genotipo x Ambiente (GGE) biplot es una herramienta de visualización de datos que muestra la interacción en una gráfica de dos vías. El GGE biplot es eficaz para el 
análisis de mega-ambientes, por ejemplo, el patrón "quien (qué genotipo)-ganó-donde (en qué localidad)", la evaluación de genotipos desde el punto de vista de estabilidad y rendimiento, y la evaluación de ambientes (poder discriminante y representatividad) [6, 7].

El objetivo de esta investigación fue estudiar el comportamiento de híbridos de maíz, por su potencial de rendimiento de grano, así como su interacción con los ambientes de evaluación, localizados en la zona tropical, mediante el análisis GGE biplot.

\section{MATERIALES Y MÉTODOS}

\section{Material genético}

Se evaluaron 14 híbridos de maíz de grano amarillo duro Tabla 1, tolerantes a suelos ácidos, que incluye variedades experimentales (híbridos simples) e híbridos triples en calidad de testigos generales.

TABLA 1. Cultivares de maíz evaluados por adaptabilidad y estabilidad a suelos ácidos en veintisiete ambientes de selección.

\begin{tabular}{|c|c|l|l|}
\hline N ${ }^{\circ}$ Cultivar & Clave & Genealogía* & \multicolumn{1}{|c|}{ Tipo } \\
\hline 1 & G1 & CLA149/CLA162 & Híbrido Simple \\
\hline 2 & G2 & CLA153/CLA167 & Híbrido Simple \\
\hline 3 & G3 & CLA155/CLA137 & Híbrido Simple \\
\hline 4 & G4 & CLA148/CLA161 & Híbrido Simple \\
\hline 5 & G5 & CLA153/CLA169 & Híbrido Simple \\
\hline 6 & G6 & CLA156/CLA138 & Híbrido Simple \\
\hline 7 & G7 & CLA7/CLA43 & Híbrido Simple \\
\hline 9 & G8 & CLA37/CLA95 & Híbrido Simple \\
\hline 10 & G9 & CLA37/CLA103 & Híbrido Simple \\
\hline 11 & G10 & CLA81/CLA111 & Híbrido Simple \\
12 & G11 & CLA87/CLA91 & Híbrido Simple \\
\hline 13 & G12 & CML359/CLA94 & Híbrido Simple \\
\hline 14 & G13 & CORPOICA H108 & Híbrido Triple \\
\hline
\end{tabular}

* CLA: Línea del CIMMYT, tolerante a suelos ácidos no liberada; CML: Línea del CIMMYT tolerante a suelos ácidos liberada; CORPOICA: Corporación Colombiana de Investigación Agropecuaria; H108 y H111 híbridos liberados por CORPOICA 2001, adaptados a suelos ácidos con buen potencial de producción.

\section{Ambientes de evaluación}

Este ensayo multiambiente fue conducido en 27 ambientes: dos en Ecuador: INIAP, Pichilingue, uno en Venezuela: Tocorón y 24 en Colombia: Caicedonia, Armenia, Pereira, Villavicencio, Villavicencio, Bucaramanga, Neiva, Palmira, Montería, Puerto López, Puerto López, El Líbano, Armenia, Garzón, Puerto López, Villavicencio, Puerto Gaitán, Puerto López, Villavicencio, Menegua, Villavicencio, Puerto Gaitán, Villavicencio, La Vizcaína Tabla 2. Los suelos de estas localidades se caracterizan por tener pH ácido (4.3 - 6.5), alta 
saturación de aluminio, bajo contenido de $\mathrm{P}, \mathrm{Ca}, \mathrm{Mg}, \mathrm{K}, \mathrm{Zn}$, B, materia orgánica, ser suelos ligeramente ondulados $(<7 \%)$ y suelos profundos.

TABLA 2. Localidades seleccionadas para evaluar estabilidad de rendimiento y adaptabilidad de 16 cultivares de maíz tolerantes a suelos ácidos en tres países de Sudamérica.

\begin{tabular}{|c|c|c|c|}
\hline \multicolumn{4}{|c|}{ Localidades } \\
\hline Clave & Estación & Zona* & País \\
\hline $\mathrm{L} 1$ & Pichilingue & Los Ríos & Ecuador \\
\hline $\mathrm{L} 2$ & Pichilingue & Los Ríos & Ecuador \\
\hline L3 & Caicedonia & Valle del Cauca, Zona Cafetera & Colombia \\
\hline L4 & Armenia & Quindío, Zona Cafetera & Colombia \\
\hline $\mathrm{L} 5$ & Pereira & Risaralda, Zona Cafetera & Colombia \\
\hline L6 & Villavicencio & Meta, Llanos Orientales & Colombia \\
\hline $\mathrm{L} 7$ & Villavicencio & Meta, Llanos Orientales & Colombia \\
\hline L8 & Bucaramanga & Santander, Valle & Colombia \\
\hline L9 & Neiva & Huila, Zona Cafetera & Colombia \\
\hline L10 & Palmira & Valle del Cauca, Valle & Colombia \\
\hline $\mathrm{L} 11$ & Montería & Córdoba, Turipaná & Colombia \\
\hline $\mathrm{L} 12$ & Tocorón & Aragua & Venezuela \\
\hline $\mathrm{L} 13$ & Puerto López & Meta, Llanos Orientales & Colombia \\
\hline $\mathrm{L} 14$ & Puerto López & Meta, Llanos Orientales & Colombia \\
\hline $\mathrm{L} 15$ & El Líbano & Tolima, Zona Cafetera & Colombia \\
\hline L16 & Armenia & Quindío, Zona Cafetera & Colombia \\
\hline $\mathrm{L} 17$ & Garzón & Huila, Zona Cafetera & Colombia \\
\hline L18 & Puerto López & Meta, Llanos Orientales & Colombia \\
\hline L19 & Villavicencio & Cauca, Zona Cafetera & Colombia \\
\hline $\mathrm{L} 20$ & Puerto Gaitán & Cauca, Zona Cafetera & Colombia \\
\hline $\mathrm{L} 21$ & Puerto López & Cauca, Zona Cafetera & Colombia \\
\hline $\mathrm{L} 22$ & Villavicencio & Meta, Llanos Orientales & Colombia \\
\hline $\mathrm{L} 23$ & Menegua & Meta, Llanos Orientales & Colombia \\
\hline $\mathrm{L} 24$ & Villavicencio & Meta, Llanos Orientales & Colombia \\
\hline $\mathrm{L} 25$ & Puerto Gaitán & Meta, Llanos Orientales & Colombia \\
\hline $\mathrm{L} 26$ & Villavicencio & Meta, Llanos Orientales & Colombia \\
\hline $\mathrm{L} 27$ & La Vizcaína & Santander Costa & Colombia \\
\hline
\end{tabular}

\section{Diseño y unidad experimental}

Se usó un diseño en bloques completos randomizados con dieciséis cultivares de maíz y tres repeticiones en cada localidad. La unidad experimental estuvo constituida por dos surcos de $5.50 \mathrm{~m}$ de longitud y $0.80 \mathrm{~m}$ de ancho, y una separación entre golpe de $0.50 \mathrm{~m}$, con tres plantas por golpe, para una densidad de población de 75 mil plantas por hectárea. 


\section{Variable de respuesta y análisis estadístico}

Se utilizaron los datos de rendimiento de grano en toneladas por hectárea (t ha ${ }^{-1}$ ) ajustados al 14\% de humedad y porcentaje de desgrane, provenientes de los ensayos de evaluación de cultivares entre los años 2006 al 2009 por el Centro Internacional de Mejoramiento de Maízy Trigo. El análisis estadístico para determinar la interacción genotipo por ambiente se realizó mediante análisis de variancia combinado y para valorar la estabilidad e interacción de los genotipos de maíz con el ambiente se utilizó el modelo GGE biplot de regresión en los sitios (SREG) [6]. En estos métodos de análisis, sólo se consideraron catorce genotipos.

El análisis de varianza combinado se lo hizo en base a un modelo mixto (ambiente aleatorio y genotipo fijo) para el rendimiento de grano bajo el modelo aditivo lineal (1)

$$
\mathrm{Y}_{\mathrm{ijk}}=\mu+\mathrm{E}_{\mathrm{k}}+(\mathrm{R} / \mathrm{E})_{\mathrm{ij}}+\mathrm{G}_{\mathrm{i}}+(\mathrm{G} * \mathrm{E})_{\mathrm{ik}}+\mathrm{e}_{\mathrm{ijk}}
$$

Donde $Y_{i j k}$ es el comportamiento medio del genotipo i en la repetición j en el ambiente $k_{i} \mu$ es la media general a través de todos los ambientes; $E_{k}$ es el efecto del ambiente $k_{\text {; }}$ $(R / E)_{i j}$ es el efecto de la repetición $j$ dentro del ambiente $k_{i} G_{i}$ es el efecto del genotipo $\left.i_{i}\left(G^{*} E\right)\right)_{i k}$ es el efecto de la interacción del genotipo $i$ en el ambiente $k_{i} e_{i j k}$ es el error experimental combinado.

En este caso, para la prueba de $\mathrm{F}$ de ambientes (E) el cuadrado medio de repeticiones/ ambientes se usó como denominador; para genotipos (G) el cuadrado medio de la IGE se usó como denominador y para la interacción (GXE) el error experimental combinado.

El GGE biplot del modelo SREG que permite la representación simultánea de la variabilidad de genotipos y ambientes, está basado en el análisis de componentes principales, cuya ecuación (2) representa el modelo básico:

$$
\bar{Y}_{i j}=\mu+\delta_{j}+\sum_{k=1} \lambda_{k} \alpha_{i k} \gamma_{j k}+\bar{\varepsilon}_{i j}
$$

Donde $Y_{i j}$ es el rendimiento promedio del genotipo $i$ en el ambiente $j_{i} \mu$ es el rendimiento promedio de todos los genotipos en el ambiente $j ; k$ es el número de componentes principales retenidos en el modelo; $\lambda k$ es el valor singular para cada componente principal; $\alpha_{i k}$ corresponde a los valores en los vectores propios de los genotipos para cada componente principal; $\gamma_{j k^{\prime}}$ son valores de los vectores propios de los ambientes para cada componente principal; $\varepsilon_{i^{\prime}}$ es el error experimental $[5,6]$.

El análisis de varianza combinado, y la prueba de significación de los componentes principales a través de la prueba de Gollob se resolvieron con el sistema SAS mientras que para GGE biplot del modelo SREG se utilizó el paquete GGEBiplotGUI del software libre R [7, 9].

\section{RESULTADOS}

\section{Análisis de varianza combinado}

El análisis de varianza combinado para rendimiento de grano Tabla 3 mostró diferencias altamente significativas $(p<0.01)$ para ambientes, genotipos y para la interacción genotipo ambiente (IGE). La alta significación observada para la IGE evidencia que los cultivares responden diferentemente en los ambientes y que esta interacción tiene gran 
magnitud. En este sentido, un genotipo que tiene una expresión estable en ensayos multiambiente contribuye poco sobre la magnitud de la IGE, destacando la necesidad de identificar esos cultivares estables con alto potencial de rendimiento [8].

TABLA 3. Análisis de la variancia para el rendimiento de grano de maíz tha-1 a través de 27 ambientes, 14 genotipos con tres repeticiones por ambiente.

\begin{tabular}{|c|c|c|c|c|c|}
\hline \multicolumn{1}{|c}{ FV } & SC & GL & CM & F & PROB \\
\hline AMBIENTES(E) & 2716.69613 & 26 & 104.48831 & 57.33420 & $<0.0001$ \\
\hline REP(AMBIENTES) & 98.41194 & 54 & 1.82244 & 2.99193 & $<0.0001$ \\
\hline GENOTIPOS(G) & 155.46435 & 13 & 11.95880 & 6.73983 & $<0.0001$ \\
\hline G ${ }^{*}$ E & 599.72970 & 338 & 1.77435 & 2.91297 & $<0.0001$ \\
\hline ERROR & 427.60246 & 702 & 0.60912 & & \\
\hline \multicolumn{1}{|r|}{ TOTAL } & 3997.90458 & 1133 & & & \\
\hline
\end{tabular}

$\mathrm{CV}=15.996 \%$

TABLA 4. Análisis de variancia y significación por la prueba de Gollob para los componentes principales en el modelo GGE biplot.

\begin{tabular}{|c|c|c|c|c|c|c|}
\hline F.V. & G.L & SC & $\mathrm{CM}$ & $\mathbf{F}$ & $\operatorname{Pr}>\mathrm{F}$ & $\%$ \\
\hline Ambientes (E) & 26 & 2716.69613 & 104.48831 & 56.5740 & & 78.2500 \\
\hline Genotipos (G) & 13 & 155.464353 & 11.95880 & 19.6370 & & 4.4800 \\
\hline GE & 338 & 599.72970 & 1.77435 & 2.9130 & & 17.2700 \\
\hline G+GE & 351 & 755.194049 & & & & \\
\hline PC1 & 38 & 279.778 & 7.36258 & 12.0896 & 0.00000 & 37.0472 \\
\hline PC2 & 36 & 139.657 & 3.87936 & 6.3700 & 0.00000 & 18.4928 \\
\hline PC3 & 34 & 91.799 & 2.69998 & 4.4335 & 0.00000 & 12.1557 \\
\hline PC4 & 32 & 55.760 & 1.74249 & 2.8612 & 0.00000 & 7.3835 \\
\hline PC5 & 30 & 47.328 & 1.57761 & 2.5905 & 0.00001 & 6.2670 \\
\hline PC6 & 28 & 35.567 & 1.27024 & 2.0858 & 0.00094 & 4.7096 \\
\hline PC7 & 26 & 27.298 & 1.04994 & 1.7240 & 0.01443 & 3.6147 \\
\hline PC8 & 24 & 22.371 & 0.93215 & 1.5306 & 0.05059 & 2.9624 \\
\hline PC9 & 22 & 18.189 & 0.82676 & 1.3576 & 0.12689 & 2.4085 \\
\hline PC10 & 20 & 14.592 & 0.72958 & 1.1980 & 0.24854 & 1.9322 \\
\hline PC11 & 18 & 11.665 & 0.64807 & 1.0641 & 0.38494 & 1.5447 \\
\hline PC12 & 16 & 7.503 & 0.46895 & 0.7700 & 0.72062 & 0.9935 \\
\hline PC13 & 14 & 3.686 & 0.26330 & 0.4323 & 0.96448 & 0.4881 \\
\hline PC14 & 12 & 0.000 & 0.00000 & 0.0000 & 1.00000 & 0.0000 \\
\hline Error & 702 & 427.602 & 0.60912 & & & \\
\hline
\end{tabular}

\section{Análisis GGE biplot del modelo SREG, contribución de los factores a la variabilidad total}

El análisis GGE biplot permitió conocer la contribución de cada fuente de variación en la partición de la suma de cuadrados total: cultivares (4.5\%), ambientes (78.2\%) e interacción 
genotipo x ambiente (17.3\%). Estos resultados coinciden con los mencionados por Salas et.al., quienes afirman que en ensayos multiambientes normales, el ambiente contribuye alrededor del $80 \%$ de la variación y que genotipos y la interacción, cada uno lo hace con aproximadamente con el 10\% de la variabilidad total Tabla 4 [9].

\section{Significación de las componentes principales}

Los dos primeros componentes principales, CP1 y CP2, fueron altamente significativos $(p<0.01)$ y explicaron el $55.54 \%$ del modelo GGE (CP1=37.05\% y PC2=18.49\%) [10]. Esta significación permitió una interpretación confiable de la IGE al usar los dos componentes principales, tanto para genotipos como para ambientes, para obtener el GGE biplot Tabla 4.

\section{Correlación entre las medias de rendimiento y las puntuaciones $\mathrm{CP} 1$ para genotipos}

La correlación alta $(r=0.85, p<0.01)$ entre las medias de los genotipos y las CP1 Tabla 4, permitió interpretar el GGE biplot, señalando que los cultivares con puntuaciones de CP1 altos se interpretaron como aquellos que tienden a tener mayores rendimientos y los ambientes con CP1 altos y CP2 cercanos a cero como aquellos ambientes que facilitan la identificación de dichos cultivares [5, 12].

FIGURA 1A. Estabilidad de 14 genotipos de maíz (G) evaluados en 27 ambientes (L), representados en el plano de Genotipo y Genotipo x Ambiente (GGE biplot), indicando el ordenamiento de genotipos basados en el rendimiento medio y estabilidad obtenido para cada ambiente.

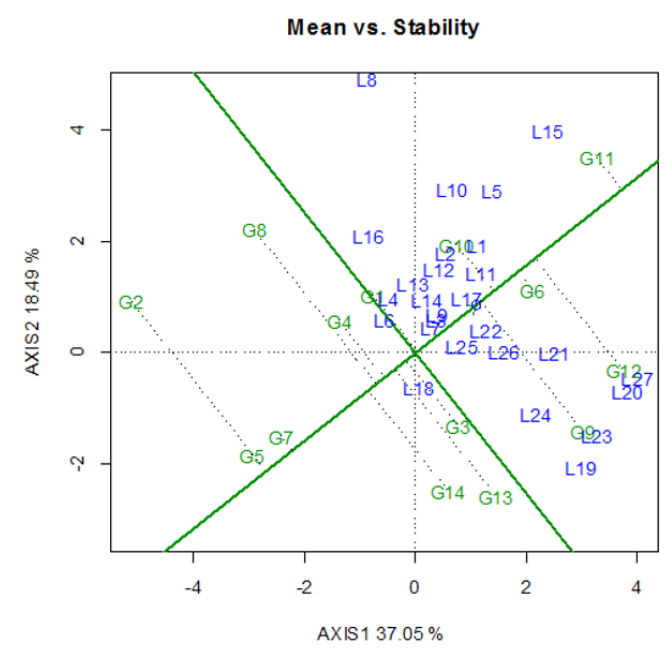

\section{Evaluación de genotipos en base al GGE biplot, rendimiento medio y estabilidad de los genotipos}

El eje de AEC (Average Environment Coordinate) o AEA (Average-Environment Axis) es la recta que pasa por origen del biplot y el ambiente promedio (pequeño círculo, que tiene como coordenadas a las medias de CP1 Y CP2 para ambientes). Fig.1 A permite conocer el rendimiento medio y la estabilidad de los genotipos $[6,7]$. Los genotipos aparecen 
ordenados por sus rendimientos medios a lo largo del eje con la flecha apuntando al mayor rendimiento. El genotipo G11 fue el cultivar con mayor rendimiento medio y estabilidad en la región estudiada, seguido del G12, G9, G6 y G10.

\section{Ordenamiento de genotipos con respecto al genotipo ideal}

Un genotipo ideal debe tener tanto alto rendimiento medio y alta estabilidad en los entornos. Fig. 1B define un genotipo "ideal" (el centro de los círculos concéntricos) como un punto del eje AEA ("absolutamente estable") en la dirección positiva y tiene una longitud de vector igual al vector más largo de los genotipos en el lado positivo de AEA ("mayor rendimiento medio"). Los genotipos situados más cerca del "genotipo ideal" son más deseables que otros. El genotipo G11 fue el más deseable, seguido de G6, luego con igual deseabilidad G12, G10 y G9 [6, 7].

FIGURA 1B. Estabilidad de 14 genotipos de máí (G) evaluados en 27 ambientes (L), representados en el plano de Genotipo y Genotipo x Ambiente (GGE biplot), indicando el ordenamiento de genotipos con respecto al genotipo ideal.

\section{Ranking Genoty pes}

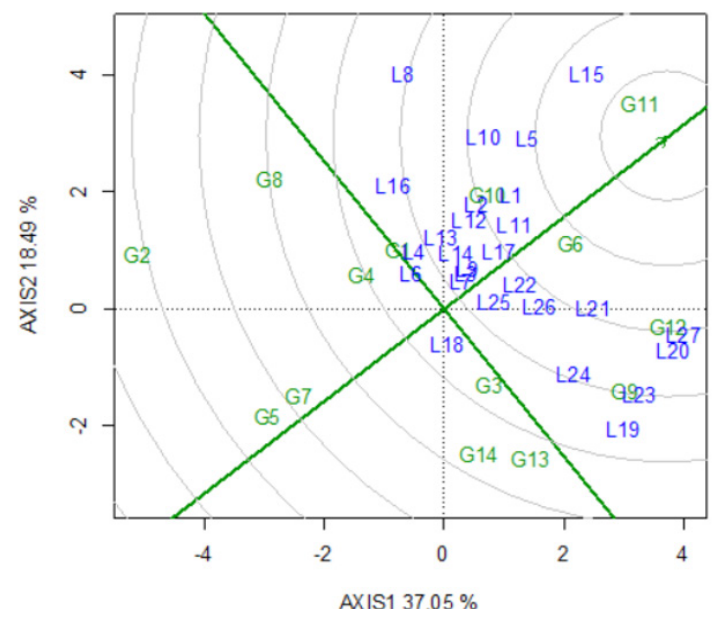

\section{Genotipos de mayor rendimiento considerando localidades -} Qué genotipo ganó y dónde

El GGE biplot Fig.1 F mostró un polígono de ocho lados formado por la unión de ocho genotipos (genotipos vértices o genotipos marcadores) los cuales son G11, G8, G2, G5, G14, G13, G9 y G12, que se encuentran más alejados del origen del biplot y tienen los vectores más largos que es la medida de la capacidad de respuestas a los ambientes en sus direcciones respectivas, de manera que todos los otros cultivares se encuentran dentro del polígono. Estos genotipos vértices fueron los que más aportan a la interacción, es decir, los de más alto y más bajo rendimiento. Los cultivares de mejor respuesta fueron G11, G12 y G9, que fueron cultivo vértices y debido a que están en la misma dirección de los ambientes. Los G2 y G5 fueron las de menor respuesta, debido a que están en una dirección opuesta a los ambientes [6,7]. 


\section{Evaluación del medio ambiente basada en GGE biplot, capacidad de discriminación de los entornos de prueba}

La longitud del vector ambiente informa sobre la utilidad del entorno de prueba para la discriminación de cultivares Fig.1 C, a mayor longitud mayor capacidad de información sobre los genotipos (discriminación) [6,7]. Los entornos de prueba L7, L3, L14, L25 y L6 por tener vectores ambiente cortos, fueron poco útiles para la discriminación de genotipos, proporcionaron poca información sobre los genotipos y por lo tanto no deberían utilizarse como entornos de prueba. Los ambientes L8, L15, L27, L20, L23 y L19 tuvieron su vector ambiente más largos, informando que estos entornos discriminan adecuadamente la variedad con vector genotipo largo en la misma dirección, tal es el caso del cultivar G11 y la L15.

FIGURA 1C. Plano de Genotipo y Genotipo x Ambiente (GGE biplot) indicando el poder discriminante de 27 ambientes (L) de evaluación para medir estabilidad de híbridos de maíz a suelos ácidos.

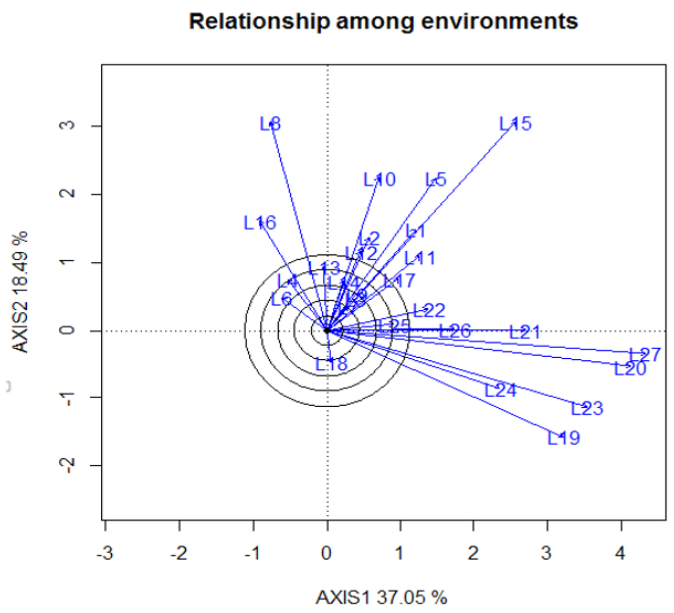

\section{Representatividad de los ambientes de prueba}

Los círculos concéntricos Fig. 1 D ayudan a visualizar la longitud de los vectores ambientes y su ángulo con el eje denominado AEA. El pequeño círculo, en cuyo centro va la dirección de la flecha, representa al "ambiente medio" y se lo obtiene promediando las coordenadas de todos los entornos de prueba (promedio de CP1 y promedio CP2 da el punto en el centro de este círculo). El eje AEA es la recta que pasa por el origen del biplot y el ambiente medio. Los ambientes de prueba que forman un ángulo más pequeño (agudo) con el eje AEA son los más representativos que el resto de los ambientes de prueba $[6,7]$. Los ambientes de prueba más representativos fueron L17, L11, L15, L1, L22 y L5, mientras que L18 y L6 fueron los menos representativos. 
FIGURA 1D. Plano de Genotipo y Genotipo x Ambiente (GGE biplot) indicando la representatividad de 27 ambientes (L) de evaluación de estabilidad de 14 genotipos de maíz $(\mathrm{G})$ a suelos ácidos.

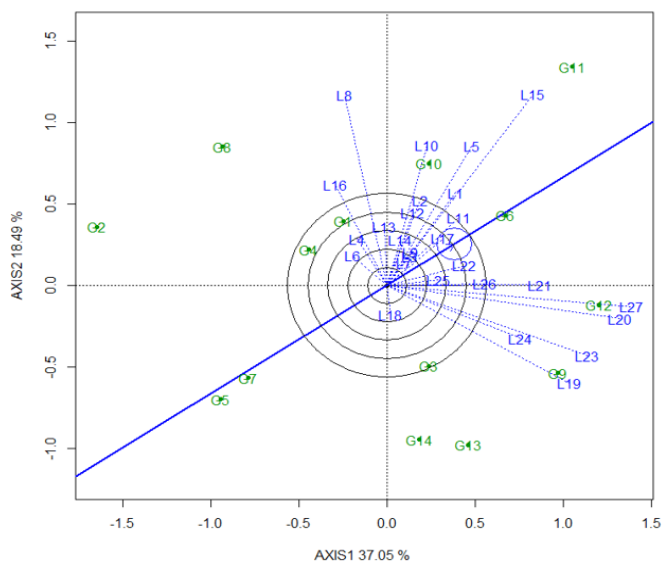

\section{Ambientes de prueba ideales para seleccionar genotipos de adaptación amplia}

Un ambiente de prueba ideal es aquel que es el más discriminante (informativo) y también el más representativo del ambiente destino [6,7]. Fig.1 E define gráficamente un ambiente de prueba ideal, el cual es el centro de los círculos concéntricos. Es un punto sobre el AEA en la dirección positiva (el más representativo) con una distancia al origen del biplot igual al vector ambiente más largo de todos los ambientes (el más informativo) [6,7]. L15-El Líbano y L5-Pereira, fueron los más cercanos a este punto, por lo que fueron los mejores, mientras que L6 y L18 fueron los más pobres para seleccionar cultivares adaptados a toda la región.

FIGURA 1E. Plano de Genotipo y Genotipo x Ambiente (GGE biplot), indicando representatividad y discriminación de 27 ambientes (L) de evaluación de la estabilidad de híbridos de maíz a suelos ácidos.

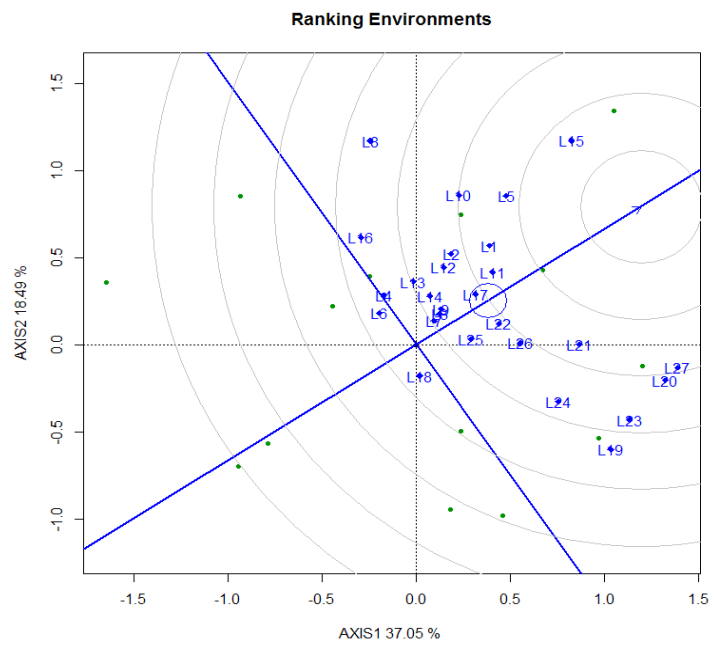




\section{Identificación de mega-ambientes}

El polígono que se muestra en Fig.1 F no solo informa qué cultivo es mejor en cada entorno de prueba, sino que también muestra dividido a los ambientes en grupos que se les Ilama mega-ambientes. Se aprecia que las líneas, que son perpendiculares a cada uno de sus lados, subdivide al polígono en siete sectores o mega-ambientes, formando grupos de sitios y cultivares. El cultivar situado en la esquina del sector es el que tiene la más alta respuesta en el sitio o sitios de este sector. Para maximizar la expresión del potencial de productividad de los cultivares, cuando existen regiones heterogéneas para la producción, a menudo es conveniente subdividir una región con ambientes heterogéneos en varios mega-ambientes relativamente homogéneos, con el objetivo de seleccionar cultivares adaptadas para cada mega-ambiente $[8,10]$.

FIGURA 1F. Mega-ambientes de evaluación de estabilidad y adaptabilidad a suelos ácidos de 14 genotipos de maíz (G) evaluados en 27 ambientes (L), representados en el Plano de Genotipo y Genotipo x Ambiente (GGE biplot).

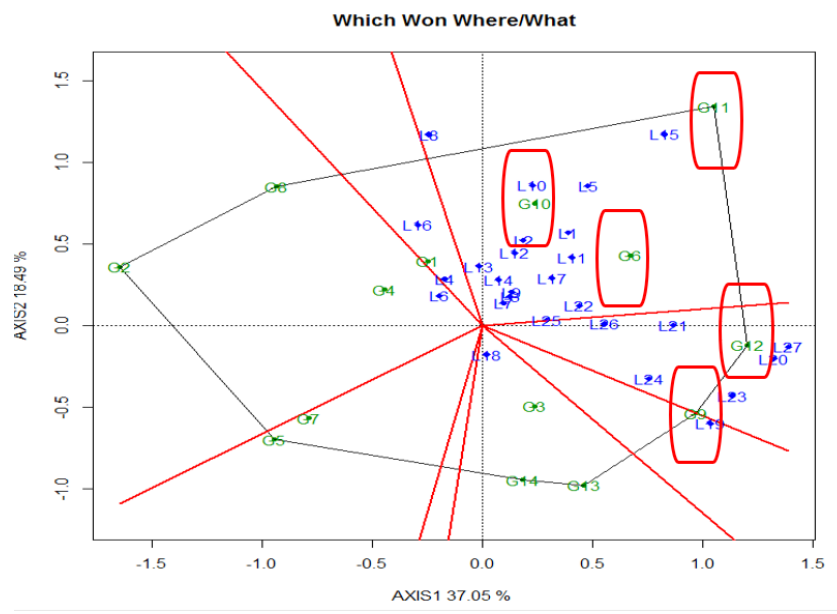

\section{DISCUSIÓN}

Para una efectiva utilización de los recursos vegetales en programas de mejora genética existe la necesidad de evaluar y describir sus variaciones fenotípicas al ser cultivados en diferentes ambientes. El problema se complica porque numerosos caracteres se heredan cuantitativamente y son muy influenciados por el ambiente, por lo que resulta necesario conseguir información sobre la Interacción Genotipo Ambiente (IGE), es decir, las diferencias genéticas entre variedades o poblaciones y sus respuestas en diversos ambientes [8].

Desde el punto de vista de la mejora de plantas, la IGE representa, un continuo reto: puede ser una eficaz ayuda, pero también puede reducir los progresos de la selección y los rendimientos esperados por el destinatario de todos los programas de mejora: el agricultor $[6,8$.$] . Por otro lado, una falta de IGE puede significar (i) falta de diversidad$ 
genética, lo que puede ser desastroso si está asociado a vulnerabilidad genética de un cultivo a enfermedades, infestaciones de insectos u otros factores; (ii) homogeneidad de los ambientes donde se prueban los genotipos [5].

La IGE significativa Tabla 3 indica que los genotipos no se comportaron de igual manera ante las variaciones ambientales de las diferentes localidades $y$, por ende, existen buenos materiales para unos ambientes pero que no responden bien en otros. La alta significación observada para ambientes ratifica la variabilidad ambiental y su significancia indica que al menos una de las localidades fue diferente al resto, atribuido a las diferentes altitudes, longitudes y latitudes en donde se ubican los ensayos; a las diferentes condiciones edafológicas, de temperatura, precipitaciones y prácticas de manejo particulares de cada una de ellas. Estas diferencias son más notorias si los ensayos multiambientes- EMA son conducidos en áreas grandes, como el presente trabajo de investigación. Estos resultados demuestran la existencia de heterogeneidad ambiental y también indican diferencias significativas entre los genotipos, ya que sus respuestas no fueron coincidentes en los entornos de prueba.

La IGE puede ocurrir debido a factores bióticos y abióticos, tales como condiciones de nutrientes y micro flora del suelo, la temperatura, incidencia de lluvias y sequía, pH, subsuelo y los factores socio-económicos que dan lugar a la aplicación de diferentes actividades de manejo en el cultivo [7, 12]. En presencia de una IGA significativa, las medias genotípicas no son la mejor representación para caracterizar el comportamiento de los genotipos ensayados sobre todos los ambientes en la identificación de genotipos superiores $[5,6,10]$.

Las magnitudes relativas de los términos de variancia correspondientes a los efectos de genotipo (G), de ambiente (A) y de interacción genotipo ambiente (IGE) Tabla 4 evidencian que el efecto atribuido al ambiente fue la fuente de variación del rendimiento de maíz más importante, explicando el 78.25\% de la suma de los efectos G + E + IGE, lo que justificaría la aplicación del GGE biplot para la evaluación de cultivares a través de ensayos multiambientales. Por otro lado, el mayor efecto relativo de IGE (17.27\%) con respecto a $\mathrm{G}(4.48 \%)$ sugiere la posible existencia de subgrupos de ambientes con capacidades discriminatorias similares (mega-ambientes) $[6,10,12]$.

El GGE biplot se utiliza, en especial, cuando el ambiente (E) es la fuente de variación más importante en relación con la contribución del genotipo (G) y la interacción genotipoambiente (IGE), como lo es en el presente trabajo de investigación. Este modelo, que incluye en el término bilineal $G+G E$, contiene los efectos del genotipo $G$ más los efectos de la IGE, de ahí que se le haya denominado como "GGE biplot"y proporciona un análisis gráfico del comportamiento (rendimiento y estabilidad) de los genotipos. Este gráfico permite identificar el genotipo de mayor potencial en cada ambiente y agrupar genotipos y ambientes con patrones similares de respuesta $[5,6,10]$.

Los dos primeros componentes principales Tabla 3 explican significativamente el 55.5\% de la variación, los cuales se representan en el "GGE biplot" que exhibe los patrones de respuesta. Generalmente, los gráficos "GGE biplot" se confeccionan utilizando los dos primeros componentes principales (CP1 y CP2), derivados en este caso, de la descomposición de los efectos combinados de G + IGE. La primera componente, CP1, 
cuando se encuentra altamente correlacionada con el efecto principal de genotipo, representa la proporción del rendimiento que se debe solo a las características del genotipo. La segunda componente, $\mathrm{CP} 2$, representa la parte del rendimiento debida a la interacción genotipo ambiente $[5,6]$. Con estos gráficos, los genotipos pueden ser evaluados en ambientes individuales o a través de ambientes, con respecto a su comportamiento promedio, a la estabilidad y a la capacidad de adaptación general o específica $[5,6,8,12]$.

El método GGE, además del análisis de genotipos, también permite el análisis de entornos. En consecuencia, el eje PC1 indica los entornos con mayor capacidad de discriminación de genotipos, y el eje PC2, los entornos más representativos del grupo $[5,10]$. Los entornos con valores altos de PC2 contribuyen más a la interacción de GE y, por lo tanto, se recomiendan para estudios de estabilidad y adaptabilidad.

\section{AGRADECIMIENTOS}

Al Centro Internacional de Mejoramiento de Maíz y Trigo (CIMMYT) por el financiamiento y facilidades brindadas para la realización de la presente investigación y por apoyo a la organización de la XXII Reunión Latinoamericana de Maíz, donde se presentó un resumen del presente trabajo. A los colegas de los Programas de Maíz de Venezuela, Colombia y Ecuador (INIAP) que apoyaron con la realización de los ensayos.

\section{CONTRIBUCIÓN DE LOS AUTORES}

Carlos Tirado, Víctor Vásquez y Luis Narro concibieron la investigación, Luis Narro administración del proyecto, curación de los datos y asesoría, Víctor Vásquez asesoría y Carlos Tirado análisis e interpretación de los datos, producción de tablas y figuras y redacción del manuscrito. 


\section{REFERENCIAS}

[1] CIMMYT. (2001). CIMMYT 1999/2000 World maize facts and trends. Meeting world maize needs: Technological opportunities and priorities for the public sector. Recuperado de: http://repository.cimmyt.org/xmlui/ handle/10883/771?show=full

[2] Lopez-Hernandez, D., Hernandez-Hernandez, R. M., \& Brossard, M. (2005). Historia del uso reciente de tierras de las sabanas de América del Sur. Estudios de casos en sabanas del Orinoco. Interciencia-INCI, 30(10), 623-632.

[3] Paliwal, R. L., Granados, G., Lafitte, H. R., \& Violic, A. D. (2001). El Maíz en los trópicos. Organización de la Naciones Unidas para la Agricultura y Alimentación. Roma, Italia. pp 392.

[4] Narro, L. A., \& Arcos, A. L. (2010). Genetics of alluminum-induced callose formation in maize roots, a selection trait for alluminum resistance. Crop Science, 50, 1848-1853.

[5] Yan, W., Hunt, L., Sheng, Q., \& Szlavnics, Z. (2000). Cultivar evaluation and megaenvironment investigation based on GGE biplot. Crop Science, 40, 597-605.

[6] Yan, W., \& Tinker, N. A. (2006). Biplot analysis of multi-environment trial data: Principles and applications. Canadian Journal of Plant Science, 86(3), 623-645.

[7] Frutos, E., Galindo, M. P., \& Leyva, V. (2014). An interactive biplot implementation in R for modeling genotype-byenvironment interaction. Stochastic Environment esearch and Risk Assesment, 28, 1629-1641.

[8] Borém, A., Condori, M., \& Miranda, G. V. (2008). Mejoramiento de Plantas (Primera ed.). Vicosa, MG, Brasil: Editora UFV.

[9] Salas, E., Juárez, H., Giraldo, D., Amoros, W., Simon, R., \& Bonierbale, M. (2009). Modelos de análisis de estabilidad y definición de ambientes basados en GIS. Lima, Peru: CIP. p 63.

[10] Yan, W., \& Hunt, L. A. (2002). Biplot analysis of multi-environment trial data. In: Kang, M. S. ed. Quantitative Genetics, Genomics and Plant Breeding. CABI Publishing, 289-303.

[11] Gollob, H. F. (1968). A statistical model which combines features of factor analytic and analysis of variances technique. Psychometrik, 33, 73-115.

[12] Balzarini, M., Bruno, C., \& Arroyo, A. (2005). Análisis de Ensayos Agrícolas Multiambientales. Ejemplos en Info-Gen. Universidad Nacional de Córdoba. Facultad de Ciencias Agropecuarias. Estadística y Biometría. Primera Ed., p 141. 